\title{
Paired-related homeobox 1 overexpression promotes multidrug resistance via PTEN/PI3K/AKT signaling in MCF-7 breast cancer cells
}

\author{
HAOYUE LUO $^{1 *}$, SHAOBO CONG ${ }^{2 *}$, JIAOJIAO DONG $^{1}$, LITAO JIN $^{1}$, DANDAN JIANG ${ }^{1}$, \\ XINGANG WANG $^{1}$, QINGFENG CHEN $^{1}$ and FUNIAN LI ${ }^{1}$
}

\author{
${ }^{1}$ Department of Breast Disease Diagnosis, Treatment Centre, Affiliated Hospital of Qingdao University, Qingdao, \\ Shandong 266000; ${ }^{2}$ Department of Biochemistry and Molecular Biology, Medical College of Qingdao University, \\ Qingdao, Shandong 266021, P.R. China
}

Received November 25, 2019; Accepted June 19, 2020

DOI: $10.3892 / \mathrm{mmr} .2020 .11414$

\begin{abstract}
Multidrug resistance (MDR) is a major cause of disease relapse and mortality in breast cancer. Paired-related homeobox 1 (PRRX1) is associated with the epithelial-mesenchymal transition (EMT), which is involved in tumor development, including cell invasion and MDR. However, the effect of PRRX1 on MDR had not clearly established. The present study investigated the influence of PRRX1 on MDR and the underlying molecular mechanisms in MCF-7 breast cancer cells. MCF-7 cells were divided into PRRX1+ group (cells transfected with a recombinant plasmid carrying the PRRX1 gene), negative control group (cells transfected with a blank vector) and blank group (untreated cells). It was found that the relative protein and mRNA expression levels of PRRX1, N-cadherin, vimentin and P-glycoprotein were significantly higher in PRRX1-overexpressing MCF-7 cells compared with those in control cells. The half-maximal inhibitory concentration of three groups after treatment with docetaxel and cis-platinum complexes were significantly higher in PRRX1-overexpressing MCF-7 cells compared with those in control cells. Furthermore, relative PTEN expression decreased significantly and levels of phosphorylated PI3K and AKT increased substantially in PRRX1-overexpressing MCF-7 cells. Theseresultsindicated that PRRX1 overexpression
\end{abstract}

Correspondence to: Dr Funian Li, Department of Breast Disease Diagnosis, Treatment Centre, Affiliated Hospital of Qingdao University, 59 Hai'er Road, Qingdao, Shandong 266000, P.R. China

E-mail: dr_lifunian@126.com

${ }^{*}$ Contributed equally

Abbreviations: EMT, epithelial-mesenchymal transition; MDR, multidrug resistance

Key words: paired related homeobox 1, multidrug resistance, breast cancer, PTEN, PI3K may induce MDR via PTEN/PI3K/AKT signaling in breast cancer. It is highly recommended that $P R R X 1$ gene expression detection should be performed in patients with breast cancer to aid the selection of more appropriate treatments, which will lead to an improved prognosis in clinical practice.

\section{Introduction}

Breast cancer is one the most frequently diagnosed cancer types in most countries and is the leading cause of cancer-associated death in $>100$ countries (1). Chemotherapy is a major treatment approach for breast cancer. However, the development of cancer cell resistance to different drugs, known as multidrug resistance (MDR), remains a challenge of cancer treatment (2). Various cellular pathways may be involved in drug resistance, including the prevention of the intracellular accumulation of anticancer drugs via transport proteins that pump drugs out of cells (3). Ample evidence suggests that the expression of ATP-binding cassette (ABC) transporters, especially $\mathrm{P}$-glycoprotein (P-gp) encoded by ABC subfamily $\mathrm{B}$ member $1(A B C B 1)$, can confer resistance to cytotoxic and targeted chemotherapy (4-6). In vitro studies have shown that ABC transporters confer resistance to numerous drugs used to treat breast cancer, such as anthracyclines, taxanes and vinca alkaloids (7). However, the mechanisms underlying the increased expression and/or activation of P-gp in breast cancer cells remains unclear.

Paired-related homeobox 1 (PRRX1) promotes EMT in breast (8), colon (9), pancreatic (10) and gastric (11) cancer. Tumor cells undergoing EMT are characterized by increased motility and invasiveness, which promotes dissemination to distant sites and the formation of metastases. In addition, tumor cells show decreased apoptosis and increased resistance to antitumor drugs, they contribute to immunosuppression and act as cancer stem-like cells (12). However, it is not clear whether PRRX1 promotes MDR in breast cancer cells.

The present study aimed to determine the effects of PRRX1 overexpression on MDR in MCF-7 cells and the underlying mechanism of the process. 


\section{Materials and methods}

Cell culture and transient transfection. MCF-7 breast cancer cells (The Cell Bank of Type Culture Collection of the Chinese Academy of Sciences) were cultured in DMEM/high glucose (HyClone; Cytiva) supplemented with $10 \%$ fetal bovine serum (Biological Industries) at $37^{\circ} \mathrm{C}$ with $5 \% \mathrm{CO}_{2}$ and $98 \%$ relative humidity in a culture incubator. Cells were separated and inoculated in 24-well plates after reaching $80 \%$ confluence. The cells in the experimental group (referred to as the PRRX1+ group) were transfected with a recombinant plasmid $(1 \mu \mathrm{g} / \mathrm{ml})$ carrying the PRRX1 gene (pEX-3/PRRX1; Shanghai GenePharma Co., Ltd.) to generate MCF-7 cells with transient PRRX1 overexpression. Cells in the negative control group (N.C group) were transfected with an empty pEX-3 vector (GenePharma), and the blank control group (the B.C group) included untreated MCF-7 cells.

The culture medium was replaced the day before transfection, and transfection was performed using Lipofectamine $^{\circledR} 2000$ (Thermo Fisher Scientific, Inc.) reagent according to the manufacturer's instructions. The morphology and transfection efficiency were observed under an inverted fluorescence microscope (magnification, x200; Olympus Corporation) $48 \mathrm{~h}$ after transfection. A total of three fluorescence images were captured randomly, and the percentage of transfected cells were calculated using the following equation: (Number of $\mathrm{GFP}^{+}$cells/total number of cells) x100. Cells $\left(\sim 9 \times 10^{5}\right.$ cells/well) in the three groups were harvested for mRNA and protein analyses.

Reverse transcription-quantitative ( $R T-q) P C R$ assay. Total RNA was extracted from cultured MCF-7 cells from the three groups using TRIzol ${ }^{\circledR}$ reagent (Beijing Solarbio Science \& Technology Co., Ltd.). A reverse transcription kit and Trans Start Probe RT-PCR Super Mix (both purchased from Transgene SA) were used to reverse transcribe RNA into template cDNA. Transcript data were obtained using the Bio-Rad One-Step Plus system (CFX96 Touch; Bio-Rad Laboratories, Inc.). Primer sequences are shown in Table I. The relative mRNA expression levels of E-cadherin, vimentin, PTEN, MDR1 and PRRX1 were determined, and normalized to the interval reference gene GAPDH. The reaction conditions were as follows: Pre-denaturation at $95^{\circ} \mathrm{C}$ for $30 \mathrm{sec}$, followed by 40 cycles of denaturation at $98^{\circ} \mathrm{C}$ for $10 \mathrm{sec}$, annealing at $60^{\circ} \mathrm{C}$ for $30 \mathrm{sec}$ and $95^{\circ} \mathrm{C}$ for $1 \mathrm{~min}$, followed by cooling at $40^{\circ} \mathrm{C}$. TaqMan (Transgene SA) probes were used. The relative mRNA expression levels were calculated using the Pfaffl method (13).

Simple western blotting analysis. Simple western blotting was performed as described previously $(14,15)$. MCF-7 cells were lysed in radioimmunoprecipitation assay buffer (Beijing Solarbio Science \& Technology Co., Ltd.) supplemented with a protease inhibitor cocktail (Sigma-Aldrich; Merck KGaA) on ice for $1 \mathrm{~h}$ and centrifuged at $8,000 \mathrm{xg}$ for $20 \mathrm{~min}$ at $4^{\circ} \mathrm{C}$. The supernatant was collected, and the protein concentration was measured using a bicinchoninic acid assay (Beyotime Institute of Biotechnology). The protein lysate was diluted with 0.1X sample buffer (cat. no. 042-195; ProteinSimple) and the final concentration of the protein sample was adjusted
Table I. Primer sequences for PRRX1, PTEN, MDR1, $\mathrm{N}$-cadherin, vimentin and GAPDH.

\begin{tabular}{ll}
\hline Gene & \multicolumn{1}{c}{ Primer sequences $\left(5^{\prime} \rightarrow 3^{\prime}\right)$} \\
\hline PRRX1 & F: GCACAGGCGGATGAGAAC \\
& R: TCTTCTGAGTTCAGCTGGTCAT \\
FTEN & R: AGTTCCCTCAGCCGTTACCT \\
RDR1 & F: CCCATCATTGCA ATAGCAGG \\
& R: TGTTCAAACTTCTGCTCCTGA \\
N-cadherin & F: CCTTTCAAACACAGCCACGG \\
& R: TGTTTGGGTCGGTCTGGATG \\
Vimentin & F: TTGACAATGCGTCTCTGGCA \\
RAPDH & F: CGTGAGGTCAGGCTTGGAAA \\
& R: AGGGGCCATCCACAGTCTTCT
\end{tabular}

F, forward; R, reverse; PRRX1, paired-related homeobox 1; MDR1, multidrug resistance protein.

to $0.2 \mathrm{mg} / \mathrm{ml}$. The samples and biotinylated ladder were denatured at $95^{\circ} \mathrm{C}$ for $5 \mathrm{~min}$ and stored on ice. A primary antibody against PRRX1 (cat. no. ab171502) was purchased from Abcam, and primary antibodies against N-Cadherin (cat. no. 13116), Vimentin (cat. no. 5741), P-gp (cat. no. 13342), PTEN (cat. no. 9188), phosphorylated (p-)AKT (cat. no. 4060), AKT (cat. no. 4691), p-PI3K (cat. no. 4228), PI3K (cat. no. 4249) and $\beta$-actin (cat. no. 3700) were purchased from CST Biological Reagents Co., Ltd. All these primary antibodies were diluted with Antibody Diluent 2 (1:50; cat. no. 042-203; ProteinSimple). The secondary antibodies used were as follows: Anti-rabbit secondary antibody (cat. no. 042-206; ProteinSimple) and anti-mouse secondary antibody (cat. no. 042-205; ProteinSimple). The secondary horseradish peroxidase conjugate (Streptavidin-HRP; cat. no. 042-414; ProteinSimple) was provided in the detection module and was ready to use. In total, $\sim 200 \mu \mathrm{l}$ Lumino-S and $200 \mu$ l of peroxide supplied in the detection module (cat. no. DM-TP01; ProteinSimple) were mixed in a microcentrifuge tube, gently pipetted and stored on ice. All reagents were added to the wells of the plate and loaded into a Wes system (ProteinSimple) for automatic western blotting assays and a capillary cartridge was inserted into the cartridge holder. Data were analyzed using Compass (version 3.1.7 1205.1438; ProteinSimple).

Cell viability analysis. Three groups of $\mathrm{MCF}-7$ cells $(4,000$ cells/well $)$ in the logarithmic growth phase were inoculated into 96-well plates. After the cells adhered to the wall, various chemotherapeutic drugs were added. The final concentrations of docetaxel (Beijing Solarbio Science \& Technology Co., Ltd.) were $0.2,0.4,0.8,1.0$ and $1.2 \mu \mathrm{mol} / 1$ and the final concentrations of cis-platinum (Beijing Solarbio Science \& Technology Co., Ltd.) complexes were $0.5,1.0,1.5$, $2.0,2.5$ and $3.0 \mu \mathrm{mol} / 1$. Three replicate wells were set at each concentration. Cells in the B.C group were used as control 
A
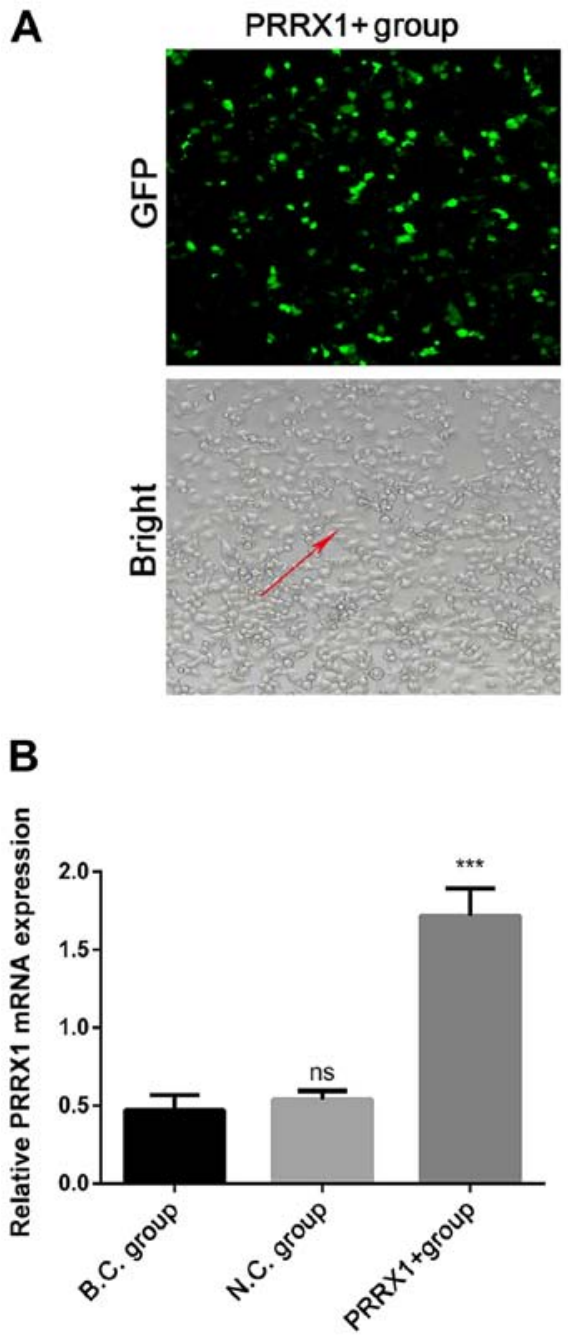
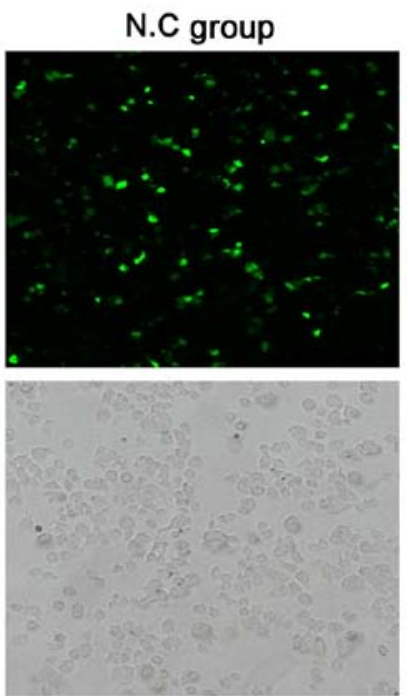

C

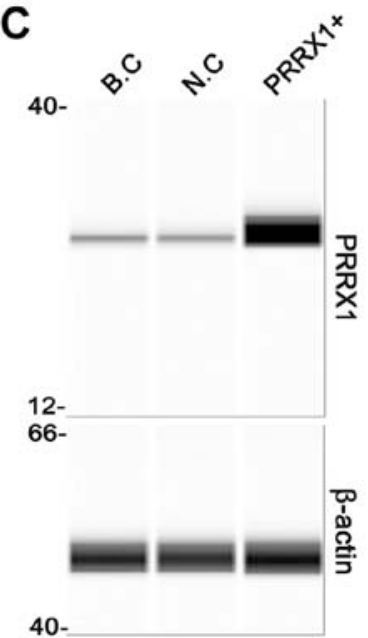

B.C group
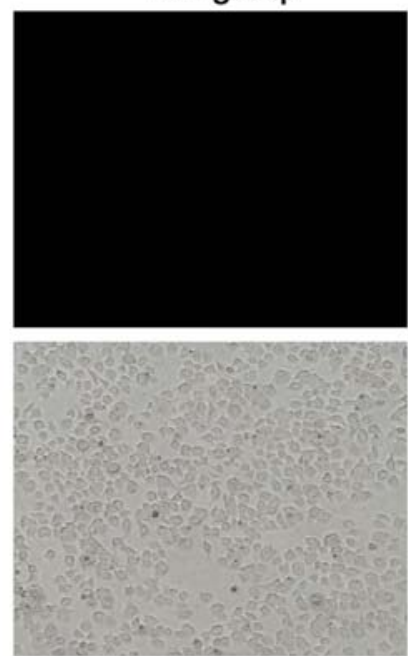

D

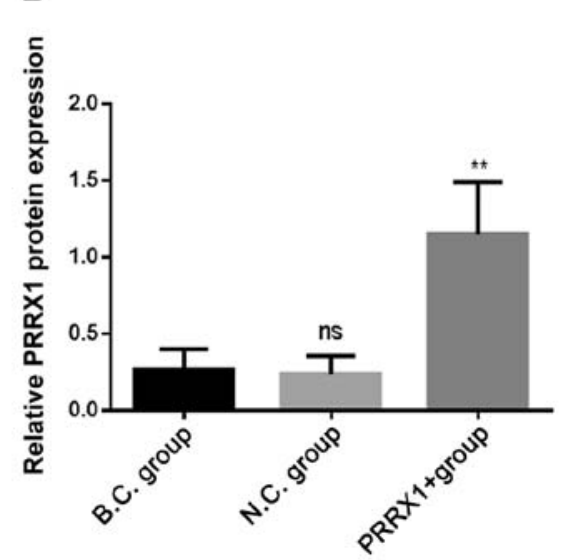

Figure 1. Transfection of MCF-7 cells with PRRX1 promotes epithelial-mesenchymal transition. (A) Morphological changes and infection efficiency were observed by inverted fluorescent microscope $48 \mathrm{~h}$ after transfection. Green fluorescent protein expression revealed that $>80 \%$ of the cells had been successfully infected with plasmids. Magnification, x200. Reverse transcription-quantitative PCR and simple western blotting were used to detect the (B) mRNA and $(\mathrm{C}$ and $\mathrm{D})$ protein expression levels of PRRX1, respectively. Values are expressed as mean \pm standard deviation. ${ }^{* *} \mathrm{P}<0.01,{ }^{* * *} \mathrm{P}<0.001$ vs. $\mathrm{BC}$ and $\mathrm{NC}$. ns, no significance; PRRX1, paired-related homeobox 1; BC, blank control; NC, negative control; ns, not significant.

wells. Blank wells without any cells were filled with equal volume (100 $\mu \mathrm{l})$ of DMEM (cat. no. 01-052-1ACS; Biological Industries). After $24 \mathrm{~h}$ of drug treatment, Cell Counting Kit (CCK)-8 (cat. no. ab228554, Abcam) reagent was added at $100 \mu \mathrm{l}$ per well and mixed, followed by incubation for $1 \mathrm{~h}$ at room temperature according to the manufacturer's protocols. Finally, the optical density (OD) at $450 \mathrm{~nm}$ was determined for each well using an enzyme-linked immunosorbent assay detection system (HBS-1096c; Nanjing Detielab Experimental Equipment Co., Ltd.). Cell viability and the half maximal inhibitory concentration $\left(\mathrm{IC}_{50}\right)$ of the two drugs in different groups were calculated using GraphPad Prism version 6 (GraphPad Software).

Statistical analysis. Data were obtained from three independent experiments and results are presented as mean \pm standard deviation. Data were analyzed using one-way ANOVA followed by Tukey's post hoc test using GraphPad Prism version 6 (GraphPad Software). $\mathrm{P}<0.05$ was considered to indicate a statistically significant difference.

\section{Results}

Transfection of MCF-7 cells with PRRX1 promotes the EMT. Cells in the three groups were observed under an inverted fluorescence microscope $48 \mathrm{~h}$ after transfection (Fig. 1A). The expression of PRRX1 and EMT markers were quantified using RT-qPCR and western blotting $48 \mathrm{~h}$ after transfection. Green fluorescent protein expression revealed that $>80 \%$ of the cells had been successfully infected. The mRNA and protein levels of PRRX1 were significantly higher in the PRRX1+ group compared with those in both control groups $(\mathrm{P}<0.001$ and $\mathrm{P}<0.01$; Fig. $1 \mathrm{~B}$ and $\mathrm{D}$, respectively). These results demonstrated that transfection was effective.

Cells in the N.C and B.C groups exhibited a paving stone shape and were tightly clustered, whereas cells in the PRRX1+ group showed a spindle morphology with loose connections among cells (bright field microscopy images, Fig. 1A). The mRNA and protein levels of N-cadherin were significantly higher in the PRRX1+ group compared with those in both control groups $(\mathrm{P}<0.05$; Fig. $2 \mathrm{~A}$ and $\mathrm{C})$. The 
A
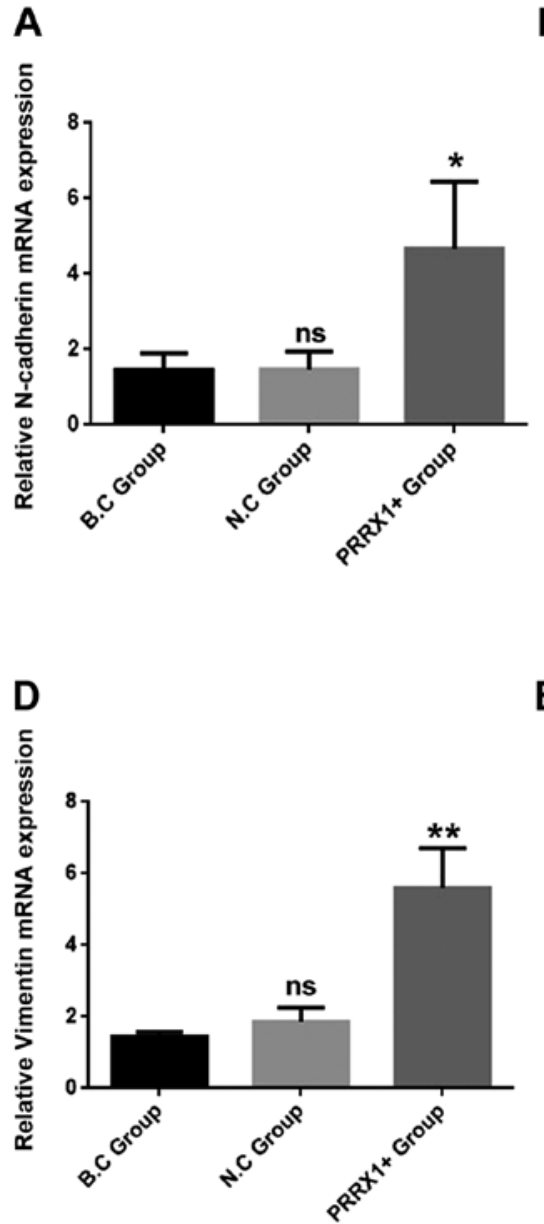

B

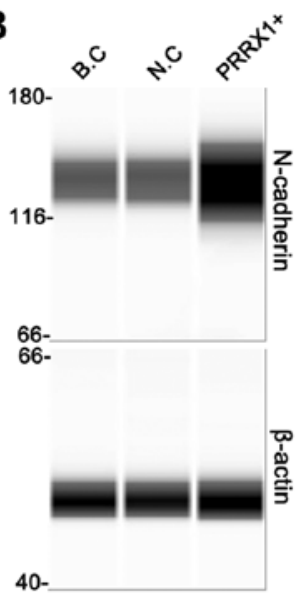

E

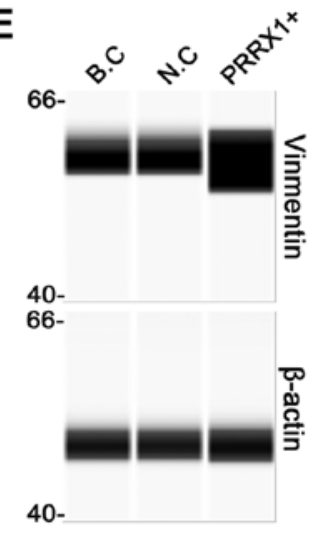

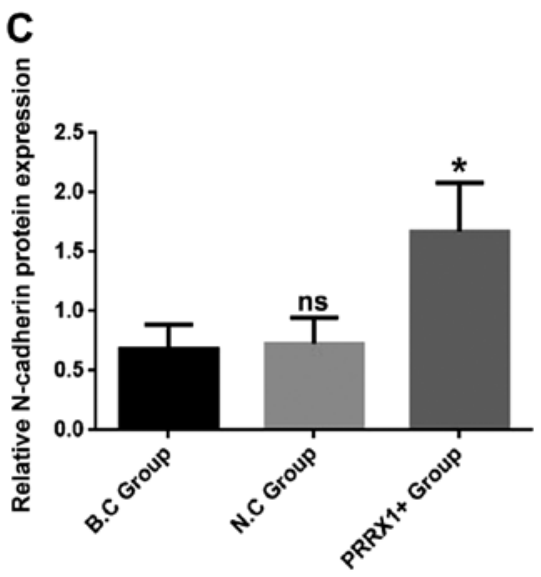

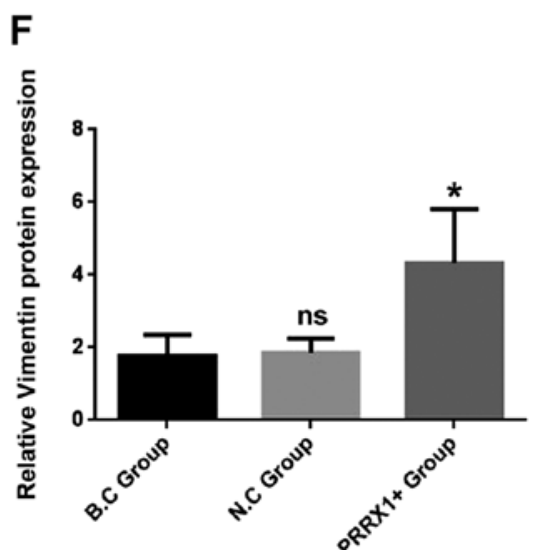

Figure 2. PRRX1 overexpression increases the expression of epithelial-mesenchymal transition-associated molecules N-cadherin and vimentin. Reverse transcription-quantitative PCR assays were used to measure the mRNA expression levels of (A) N-cadherin and (D) vimentin. Simple western blotting was used to detect the protein expression levels of (B and $\mathrm{C}) \mathrm{N}$-cadherin and ( $\mathrm{E}$ and $\mathrm{F})$ vimentin. Values are expressed as mean \pm standard deviation. ${ }^{*} \mathrm{P}<0.05$, ${ }^{* *} \mathrm{P}<0.01$ vs. BC and NC. ns, no significance; PRRX1, paired-related homeobox 1; BC, blank control; NC, negative control; ns, not significant.

mRNA and protein levels of vimentin were also significantly higher in the PRRX1+ group compared with those in both control groups $(\mathrm{P}<0.01$ and $\mathrm{P}<0.05$; Fig. $2 \mathrm{D}$ and $\mathrm{F}$, respectively). These results indicated that PRRX1 promotes EMT in breast cancer cells.

PRRX1 overexpression promotes MDR and increases $P$-gp expression. Cell proliferation was analyzed in the three groups using a CCK-8 assay after treatment with docetaxel and cis-platinum complexes for $24 \mathrm{~h}$. The $\mathrm{IC}_{50}$ values of docetaxel and cis-platinum complexes for cells transfected with PRRX1 were significantly higher compared with those of the two control groups (both $\mathrm{P}<0.01$; Fig. $3 \mathrm{~A}$ and $\mathrm{B}$, respectively). These results indicated that overexpression of PRRX1 decreased the inhibitory effects of docetaxel and cis-platinum complexes on proliferation in breast cancer cells.

To further verify the effect of PRRX1 on MDR in breast cancer, the expression levels of the MDR-associated protein P-gp were analyzed in the three groups using RT-qPCR and western blotting. As shown in Fig. $3 \mathrm{C}$ and D, P-gp mRNA and protein levels were significantly higher in the PRRX1+ group compared with those in both control groups (both $\mathrm{P}<0.01$ ). These results revealed that PRRX1 can promote $\mathrm{P}$-gp-associated MDR in breast cancer cells.
PRRX1 overexpression increases $p$-PI3K and AKT expression and decreases PTEN. The present study further evaluated the mechanisms by which PRRX1 promotes MDR by investigating the roles of PI3K and AKT. The levels of p-PI3K and p-AKT proteins (Fig. 4A and B) and the ratio of $\mathrm{p}-\mathrm{PI} 3 \mathrm{~K} / \mathrm{PI} 3 \mathrm{~K}$ and $\mathrm{p}-\mathrm{AKT} / \mathrm{AKT}$ were significantly higher in the PRRX1+ group compared with those in both control groups $(\mathrm{P}<0.01$; Fig. 4C and D), whereas the protein expression of total PI3K and AKT in the three groups showed no difference (Fig. 4A and B). It has been reported that PTEN acts as a tumor inhibitor gene by negatively regulating the PI3K/Akt pathway (16). Next, it was explored whether PRRX1 regulates the PI3K and Akt phosphorylation by downregulating PTEN expression. As shown in Fig. 4E-G, the mRNA and protein levels of PTEN were significantly lower in the PRRX1+ group compared with those in the two control groups (both $\mathrm{P}<0.01$ ). These results suggested that PRRX1 is a potential regulator of PI3K/Akt signaling pathway in breast cancer cells.

\section{Discussion}

Although several chemotherapy regiments have contributed to a marked increase in the survival rates of patients with breast cancer, MDR remains a serious issue with respect to drug 
A

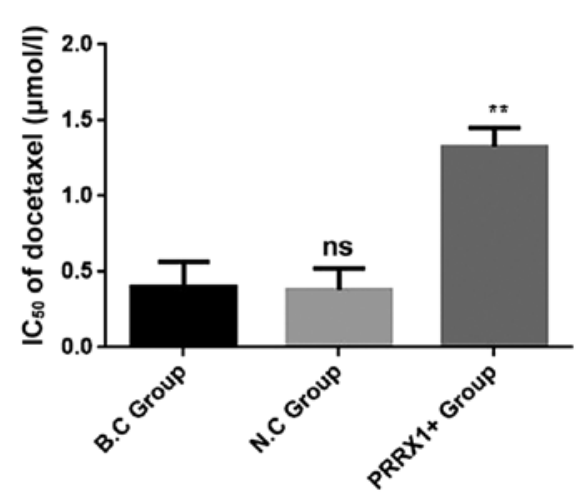

B

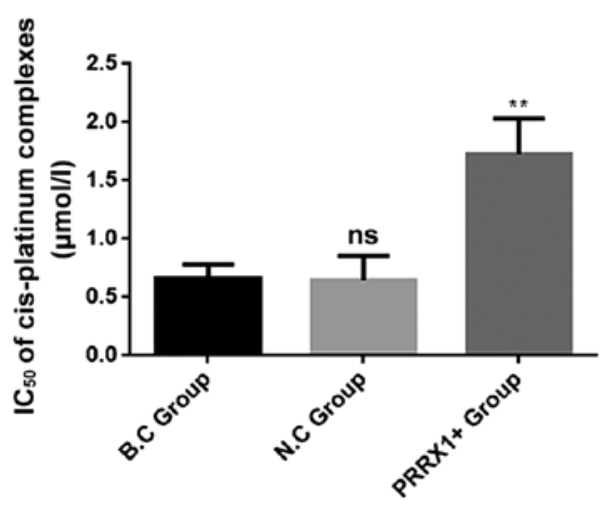

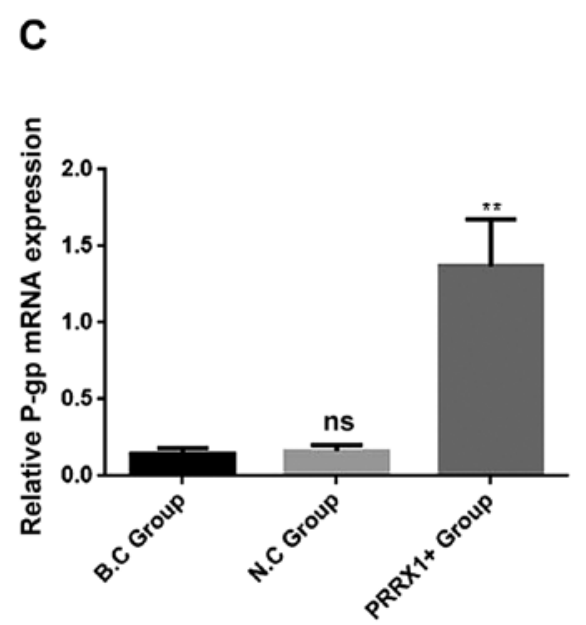

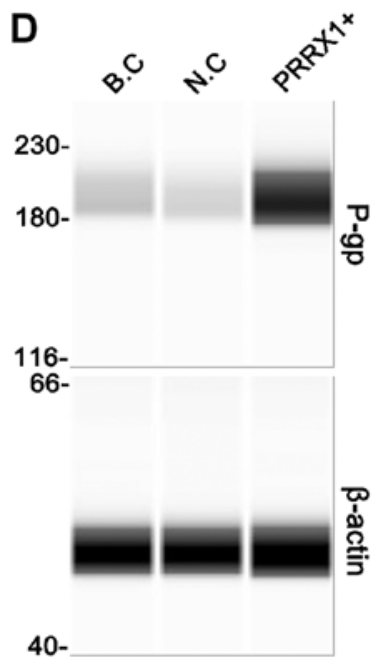

E

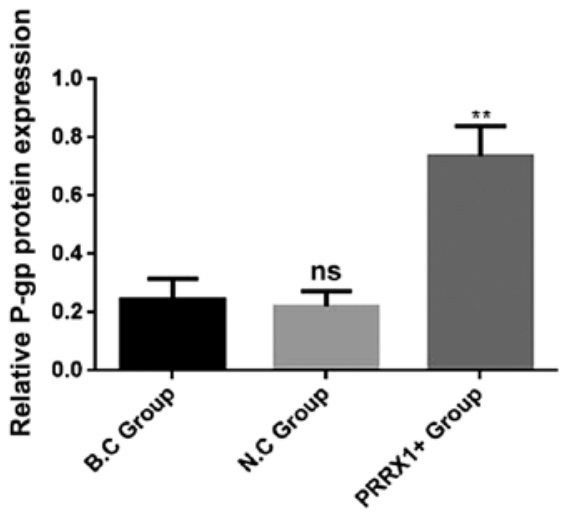

Figure 3. PRRX1 overexpression promotes multidrug resistance and increases P-gp expression. A Cell Counting Kit-8 assay was performed to detect the $\mathrm{IC}_{50}$ values of the three groups after treated with (A) docetaxel and (B) cis-platinum complexes for $24 \mathrm{~h}$. Reverse transcription-quantitative PCR assays and simple western blotting were used to detect the $(C)$ mRNA and (D and E) protein expression levels of P-gp. Values are expressed as mean \pm standard deviation. ${ }^{* *} \mathrm{P}<0.01$ vs. BC and NC. ns, no significance; PRRX1, paired-related homeobox 1; P-gp, P-glycoprotein; $\mathrm{IC}_{50}$, half maximal inhibitory concentration; ns, not significant.

efficacy (17) and is associated with the risk of relapse and poor prognosis (18). Accordingly, methods to predict and circumvent MDR are likely to improve chemotherapy outcomes.

EMT is a transitional process accompanied by changes in cell morphology along with loss of polarity, intercellular junctions and expression of the EMT markers, such as N-cadherin, E-cadherin and vimentin (19). Several studies have shown that there is an association between EMT and insensitivity to chemotherapeutic agents (20-22). Fischer et al (23) reported that EMT induces cyclophosphamide resistance by the activation of aldehyde dehydrogenase in breast cancer cells. Saxena et al (24) revealed that in addition to invasion, EMT inducers also promote drug resistance by upregulating $\mathrm{ABC}$ transporters, which efflux chemotherapeutic drugs. PRRX1 is a newly identified EMT inducer and confers invasive properties in cancer cells (8). The present results, along with the aforementioned studies, showed that MCF-7 cells undergo EMT in response to PRRX1 overexpression, exhibiting a spindle-like shape and increased expression levels of the mesenchymal markers $\mathrm{N}$-cadherin and vimentin.

The ability of PRRX1 to promote MDR in breast cancer cells has not been investigated, to the best of our knowledge. Previous studies have shown that PRRX1 promotes EMT and is associated with metastasis, poor prognosis and radiotherapy resistance in colorectal cancer $(9,25)$. However, in lung cancer cells the loss of PRRX1 induces EMT and induces antiapoptotic ability and resistance to cisplatin $(26,27)$. The present study demonstrated that PRRX1 decreases the sensitivity of MCF-7 breast cancer cells to different drugs. These data, along with the aforementioned studies, suggested that PRRX1 has distinct functions among different types of cancer. In tumor cell lines, MDR is often associated with an ATP-dependent decrease in cellular drug accumulation, which is attributed to the overexpression of certain ABC transporter proteins $(28,29)$. Among causes of MDR, the overexpression of P-gp, encoded by MDRI, plays a notable role in drug recognition and export before reaching intracellular targets (30). The present study demonstrated that MDR is increased in PRRX1-overexpressing cells and that P-gp levels in these cells are higher compared with those in both control cells. A previous study has shown that PRRX1 expression is higher in breast cancer tissues compared with adjacent normal tissues and is associated with the migration and invasion of breast cancer cells (31). The expression status of PRRX1 in patients with and without drug resistant should be analyzed in future research to further investigate the clinical significance of PRRX1 in drug resistance. 
A

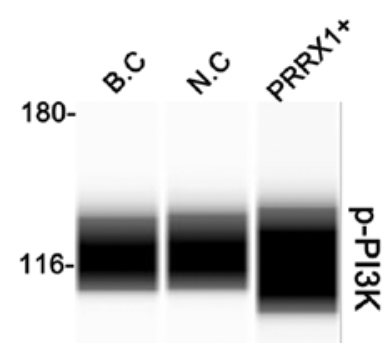

$66-$

$180-$

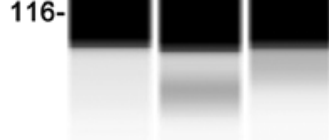

$66-$

$66-$

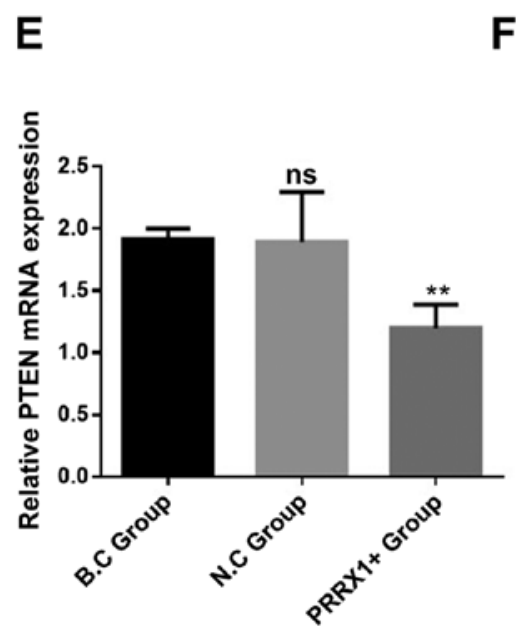

B

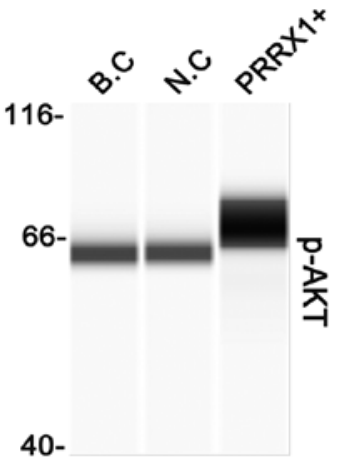

$116-$

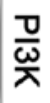

$\mathbf{F}$

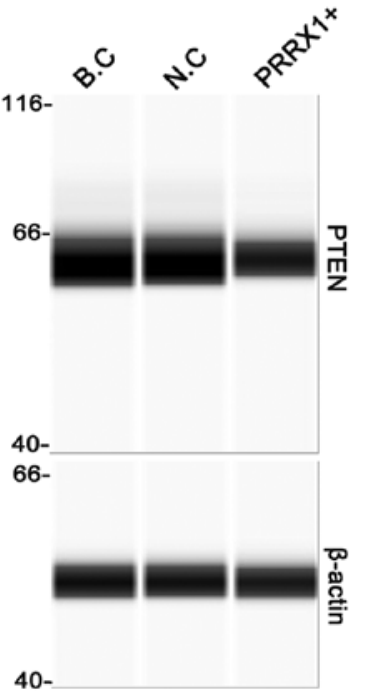

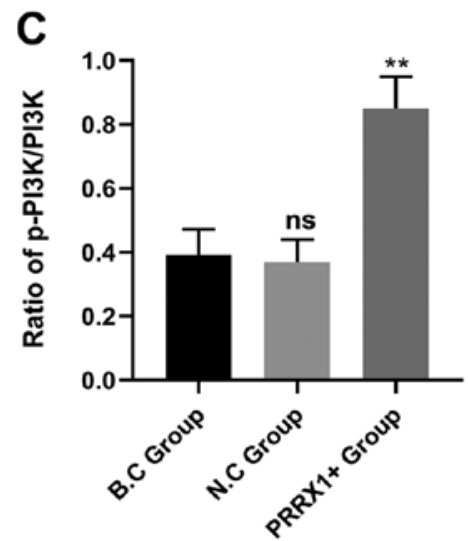

D

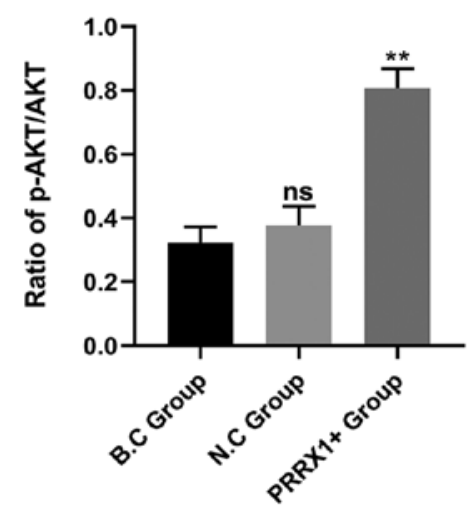

G

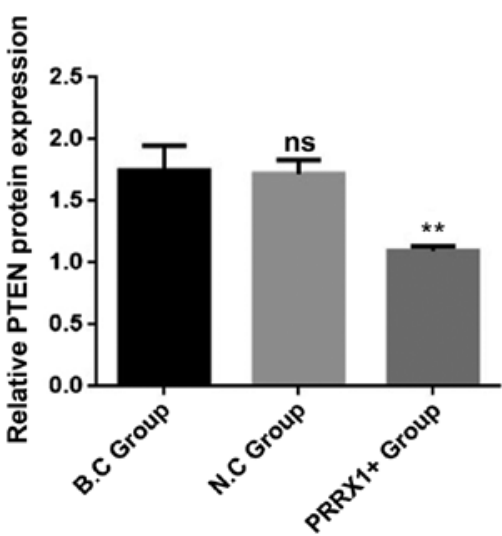

Figure 4. PRRX1 overexpression increases p-PI3K and p-AKT expression and decreases PTEN. Simple western blotting was used to detect the phosphorylation levels of (A) PI3K and (B) AKT. (C) The ratio of p-PI3K/PI3K and (D) p-AKT/AKT was calculated and presented. In addition, reverse transcription-quantitative PCR assays and simple western blotting were used to detect the (E) mRNA and (F and G) protein levels of PTEN. Values are expressed as mean \pm standard deviation. ${ }^{* *} \mathrm{P}<0.01$ vs. BC and NC; ns, no significance; PRRX1, paired-related homeobox 1; BC, blank control; NC, negative control; ns, not significant.

The PI3K/AKT signaling pathway is associated with various biochemical processes, including EMT $(32,33)$, MDR $(34,35)$, cell invasion, migration $(36,37)$ and proliferation $(38,39)$. As a key tumor suppressor gene, PTEN can inhibit the PI3K/AKT signaling pathway and serves an important role in the regulation of a series of biological processes. The loss of PTEN activity leads to uncontrolled PI3K signaling, which is found in a variety of primary and metastatic tumors, including breast cancer $(40,41)$. The present study reported that PRRX1 overexpression in MCF-7 cells resulted in a decrease in PTEN and increases in the phosphorylation of PI3K and AKT. It was also demonstrated that PRRX1 may promote MDR through the activation of the PTEN/PI3K/AKT signaling pathway. Inhibitors of PI3K and AKT will be applied in future studies to further verify the function of PI3K/AKT signaling in the PRRX1-induced MDR.

The present study is the first, to the best of our knowledge, suggesting that PRRX1 promotes MDR in breast cancer cells. However, only one cell line was used, and no drug resistant cell lines were constructed, which are limitations of the present 
study. Moreover, the reverse effect of PRRX1 downregulation on MDR and other associated pathways were not investigated. Further research is needed to evaluate drug resistance through combined detection of multiple indicators using different tumor cell lines. Nevertheless, the present findings demonstrated that PRRX1 overexpression induced the EMT process and promoted MDR in MCF-7 breast cancer cells, which may be underpinned by PTEN/PI3K/AKT signaling. The present study not only provides novel insights into the role of PRRX1 in MDR, but also highlights the potential of PRRX1 as a novel target for breast cancer treatment.

\section{Acknowledgements}

Not applicable.

\section{Funding}

This study was supported by the National Natural Science Foundation of China (grant nos. 8130-2290 and 81700029) and the Natural Science Foundation of Shandong Province (grant no. ZR-2017PH032).

\section{Availability of data and materials}

All data generated or analyzed during this study are included in this published article.

\section{Authors' contributions}

FL, HL and JD designed the study. HL and SC conducted the experiments and wrote the manuscript. LJ and DJ analyzed the data. XW and QC collected data, performed data analysis and revised the manuscript. All authors read and approved the final manuscript.

\section{Ethics approval and consent to participate}

Not applicable.

\section{Patient consent for publication}

Not applicable.

\section{Competing interests}

The authors declare that they have no competing interests.

\section{References}

1. Bray F, Ferlay J, Soerjomataram I, Siegel RL, Torre LA and Jemal A: Global cancer statistics 2018: GLOBOCAN estimates of incidence and mortality worldwide for 36 cancers in 185 countries. CA Cancer J Clin 68: 394-424, 2018.

2. Pastan I and Gottesman M: Multiple-drug resistance in human cancer. N Engl J Med 316: 1388-1393, 1987.

3. Trock BJ, Leonessa F and Clarke R: Multidrug resistance in breast cancer: A meta-analysis of MDR1/gp170 expression and its possible functional significance. J Natl Cancer Inst 89 917-931, 1997.

4. Robey RW, Pluchino KM, Hall MD, Fojo AT, Bates SE and Gottesman MM: Revisiting the role of ABC transporters in multidrug-resistant cancer. Nat Rev Cancer 18: 454-464, 2018.
5. Battistella $\mathrm{C}$ and Klok HA: Reversion of P-gp-mediated drug resistance in ovarian carcinoma cells with PHPMA-zosuquidar conjugates. Biomacromolecules 18: 1855-1865, 2017.

6. De Vera AA, Gupta P, Lei Z, Liao D, Narayanan S, Teng Q, Reznik SE and Chen ZS: Immuno-oncology agent IPI-549 is a modulator of P-glycoprotein (P-gp, MDR1, ABCB1)-mediated multidrug resistance (MDR) in cancer: In vitro and in vivo. Cancer Lett 442: 91-103, 2019.

7. Szakács G, Paterson JK, Ludwig JA, Booth-Genthe C and Gottesman MM: Targeting multidrug resistance in cancer. Nat Rev Drug Discov 5: 219-234, 2006.

8. Ocaña OH, Córcoles R, Fabra A, Moreno-Bueno G, Acloque H, Vega S, Barrallo-Gimeno A, Cano A and Nieto MA: Metastatic colonization requires the repression of the epithelial-mesenchymal transition inducer Prrx1. Cancer Cell 22: 709-724, 2012.

9. Takahashi Y, Sawada G, Kurashige J, Uchi R, Matsumura T, Ueo H, Takano Y, Akiyoshi S, Eguchi H, Sudo T, et al: Paired related homoeobox 1, a new EMT inducer, is involved in metastasis and poor prognosis in colorectal cancer. Br J Cancer 109: 307-311, 2013.

10. Reichert M, Takano S, Von Burstin J, Kim SB, Lee JS, Ihida-Stansbury K, Hahn C, Heeg S, Schneider G, Rhim AD, et al: The Prrx1 homeodomain transcription factor plays a central role in pancreatic regeneration and carcinogenesis. Genes Dev 27: 288-300, 2013.

11. Guo J, Fu Z, Wei J, Lu W, Feng J and Zhang S: PRRX1 promotes epithelial-mesenchymal transition through the Wnt/ $\beta$-catenin pathway in gastric cancer. Med Oncol 32: 393, 2015.

12. Marcucci F, Stassi G and Maria RD: Epithelial-mesenchymal transition: A new target in anticancer drug discovery. Nat Rev Drug Discov 15: 311-325, 2016

13. Pfaffl MW: A new mathematical model for relative quantification in real-time RT-PCR. Nucleic Acids Res 29: e45, 2001.

14. Fourier A, Escal J, Bernard E, Lachman I, Perret-Liaudet A, Leblanc P and Quadrio I: Development of an automated capillary nano-immunoassay-simple western assay-to quantify total TDP43 protein in human platelet samples. Anal Bioanal Chem 411: 267-275, 2019.

15. Dahl JA, Jung I, Aanes H, Greggains GD, Manaf A, Lerdrup M, Li G, Kuan S, Li B, Lee AY, et al: Broad histone H3K4me3 domains in mouse oocytes modulate maternal-to-zygotic transition. Nature 537: 548-552, 2016.

16. Maehama T, Taylor GS and Dixon JE: PTEN and myotubularin: Novel phosphoinositide phosphatases. Annu Rev Biochem 70: 247-279, 2001.

17. Videira M, Reis RL and Brito MA: Deconstructing breast cancer cell biology and the mechanisms of multidrug resistance. Biochim Biophys Acta 1846: 312-325, 2014.

18. Kovalchuk O, Filkowski J, Meservy J, Ilnytskyy Y, Tryndyak VP, Chekhun VF and Pogribny IP: Involvement of microRNA-451 in resistance of the MCF-7 breast cancer cells to chemotherapeutic drug doxorubicin. Mol Cancer Ther 7: 2152-2159, 2008.

19. Zeisberg $M$ and Neilson EG: Biomarkers for epithelialmesenchymal transitions. J Clin Invest 119: 1429-1437, 2009.

20. Mallini P, Lennard TWJ, Kirby JA and Meeson A: Epithelial-to-mesenchymal transition: What is the impact on breast cancer stem cells and drug resistance. Cancer Treat Rev 40: 341-348, 2014.

21. Rice AJ, Cortes E, Lachowski D, Cheung BCH, Karim SA, Morton JP and Del Río Hernández A: Matrix stiffness induces epithelial-mesenchymal transition and promotes chemoresistance in pancreatic cancer cells. Oncogenesis 6: e352, 2017.

22. Zheng X, Carstens JL, Kim J, Scheible M, Kaye J, Sugimoto H, Wu CC, LeBleu VS and Kalluri R: Epithelial-to-mesenchymal transition is dispensable for metastasis but induces chemoresistance in pancreatic cancer. Nature 527: 525-530, 2015.

23. Fischer KR, Durrans A, Lee S, Sheng J, Li F, Wong ST, Choi H, El Rayes T, Ryu S, Troeger J, et al: Epithelial-to-mesenchymal transition is not required for lung metastasis but contributes to chemoresistance. Nature 527: 472-476, 2015.

24. Saxena M, Stephens MA, Pathak H and Rangarajan A: Transcription factors that mediate epithelial-mesenchymal transition lead to multidrug resistance by upregulating $\mathrm{ABC}$ transporters. Cell Death Dis 2: e179, 2011.

25. Lin SM, Xia Q, Zhang YQ, Sun AM and Chen LH: miR-124 regulates radiosensitivity of colorectal cancer cells by targeting PRRX1. Nan Fang Yi Ke Da Xue Xue Bao 36: 1110-1116, 2016 (In Chinese).

26. Zhu H and Sun G: Loss of PRRX1 induces epithelial-mesenchymal transition and cancer stem cell-like properties in A549 cells. Am J Transl Res 9: 1641-1650, 2017. 
27. Zhu H, Sun G, Dong J and Fei L: The role of PRRX1 in the apoptosis of A549 cells induced by cisplatin. Am J Transl Res 9: 396-402, 2017

28. Leonard GD, Fojo T and Bates SE: The role of ABC transporters in clinical practice. Oncologist 8: 411-424, 2003.

29. Schinkel AH and Jonker JW: Mammalian drug efflux transporters of the ATP binding cassette (ABC) family: An overview. Adv Drug Del Rev 55: 3-29, 2003.

30. Meng H, Liong M, Xia T, Li Z, Ji Z, Zink JI and Nel AE: Engineered design of mesoporous silica nanoparticles to deliver doxorubicin and P-glycoprotein siRNA to overcome drug resistance in a cancer cell line. ACS Nano 4: 4539-4550, 2010.

31. Lv Z, Kong B, Liu X, Jin LY, Dong Q, Li FN and Wang HB miR-655 suppresses epithelial-to-mesenchymal transition by targeting Prrx1 in triple-negative breast cancer. J Cell Mol Med 20: 864-873, 2016.

32. Perumal E, Youn KS, Sun S, Seung-Hyun J, Suji M, Jieying L and Yeun-Jun C: PTEN inactivation induces epithelial-mesenchymal transition and metastasis by intranuclear translocation of $\beta$-catenin and snail/slug in non-small cell lung carcinoma cells. Lung Cancer 130: 25-34, 2019.

33. Yan R, Wang Y, Shi M, Xiao Y, Liu L, Liu L and Guo B: Regulation of PTEN/AKT/FAK pathways by PPAR $\gamma$ impacts on fibrosis in diabetic nephropathy. J Cell Biochem Jan 16, 2019 (Epub ahead of print).

34. Gao C, Yuan X, Jiang Z, Gan D, Ding L, Sun Y, Zhou J, Xu L, Liu Y and Wang G: Regulation of AKT phosphorylation by GSK3 $\beta$ and PTEN to control chemoresistance in breast cancer. Breast Cancer Res Treat 176: 291-301, 2019.
35. Wang L, Lin $\mathrm{N}$ and $\mathrm{Li}$ Y: The PI3K/AKT signaling pathway regulates ABCG2 expression and confers resistance to chemotherapy in human multiple myeloma. Oncol Rep 41: 1678-1690, 2019.

36. Zhang Y, Sui R, Chen Y, Liang H, Shi J and Piao H: Long noncoding RNA MT1JP inhibits proliferation, invasion, and migration while promoting apoptosis of glioma cells through the activation of PTEN/Akt signaling pathway. J Cell Physiol 234: 19553-19564, 2019.

37. Chen SR, Cai WP, Dai Xj, Guo AS, Chen HP, Lin GS and Lin RS: Research on miR-126 in glioma targeted regulation of PTEN/PI3K/Akt and MDM2-p53 pathways. Eur Rev Med Pharmacol Sci 23: 3461-3470, 2019.

38. Feng H, Zhang Z, Qing X, French SW and Liu D: miR-186-5p promotes cell growth, migration and invasion of lung adenocarcinoma by targeting PTEN. Exp Mol Pathol 108: 105-113, 2019.

39. Wang R, Wang F, Cao $\mathrm{H}$ and Yang JY: miR-223 regulates proliferation and apoptosis of IL-22-stimulated HaCat human keratinocyte cell lines via the PTEN/Akt pathway. Life Sci 230: 28-34, 2019.

40. Engelman JA, Luo J and Cantley LC: The evolution of phosphatidylinositol 3-kinases as regulators of growth and metabolism. Nat Rev Genet 7: 606-619, 2006.

41. Shaw RJ and Cantley LC: Ras, PI(3)K and mTOR signalling controls tumour cell growth. Nature 441: 424-430, 2006.

(c) (i) () $९$ This work is licensed under a Creative Commons

EY NG ND Attribution-NonCommercial-NoDerivatives 4.0 International (CC BY-NC-ND 4.0) License. 